\title{
Injury and illness profiles during the 2014 South African Ironman triathlon
}

L J Holtzhausen, ${ }^{\mathbf{M}} \mathrm{MBChB}$, MPhil (Sports Medicine), PhD, FACSM, C R Smit, ${ }^{1}$ MBChB, M (Sports Medicine), G Joubert, ${ }^{2}$ $\mathrm{BA}, \mathrm{MSc}$, K von Hagen, ${ }^{3} \mathrm{MBChB}, \mathrm{MSc}$ (Sports Medicine)

${ }^{1}$ Department of Sport and Exercise Medicine, Faculty of Health Sciences, University of the Free State, Bloemfontein, South Africa

2 Department of Biostatistics, Faculty of Health Sciences, University of the Free State, Bloemfontein, South Africa

${ }^{3}$ Intercare Walmer, Kings Court Mall, Corner Buffelsfontein and Titian Roads, Port Elizabeth, South Africa

\section{Corresponding author: L J Holtzhausen (holt.louis@gmail.com)}

Background: There is a need for ongoing scrutiny of injury and illness profiles of ultra-distance athletes. This study aimed to record the medical history, illness and injuries of athletes receiving medical attention during the 2014 Ironman South Africa (IMSA) triathlon, and to investigate the temporal presentation of medical encounters.

Methods: This was a retrospective, cross-sectional study. All athletes who required medical attention at the main medical tent and all of the medical posts or mobile units along the route were included in this study A total of 2331 athletes started the race. Data included age, gender, time and stage of the race when medical attention was required, pre-race medical history and medication use, illness and injuries treated, special investigations performed, and weather conditions.

Results: Overall, 179 athletes $(7.7 \%)$ required medical attention. The incidence of medical encounters was $7.8 \%$. A significantly higher percentage of younger participants encountered medical problems $(P=0.04)$. Most patient encounters $(80.1 \%)$ occurred after the race. The median duration of treatment was 26 minutes. Medication was used by $35.1 \%$ of patients during the race. The most common medical encounters were exertion-related $(71.2 \%)$, gastrointestinal $(16.4 \%)$, dermatological $(11.9 \%)$, musculoskeletal $(9.6 \%)$ and cardiorespiratory conditions (2.4\%).

Conclusion: Medical encounters occurred more frequently in later stages of the race. Most medical conditions were exertionrelated. Potential higher risk may be associated with medication use, recent illness, and in younger participants. Temporal stacking of medical personnel, planning of resources according to expected conditions, preventative measures for high-risk behaviour, and on-going data collection with comparable methodology are recommended. Keywords: triathletes, medical encounters, medication use, treatment, event medical management

\section{S Afr J Sports Med 2018;30:1-6. DOI: 10.17159/2078-516X/2018/v30i1a1509}

The Ironman South Africa (IMSA) is one of 28 global Ironman triathlon races and combines the sports of open water swimming $(3.8 \mathrm{~km})$, cycling $(180 \mathrm{~km})$ and running $(42.2 \mathrm{~km})$. Triathletes are exposed to more challenging environmental conditions and physiological demands than athletes participating in most other individual sporting events. The long duration and extreme physical and mental demands of the sport place severe strain on participants. Triathletes may consequently present with a variety of medical problems during the competition, including exhaustion and dehydration, muscle cramping, hypothermia, heat stroke and musculoskeletal injuries. ${ }^{[1,2,3]}$

In most triathlon events, the swimming segment is potentially the most dangerous due to complications such as, accidental drowning, hypothermia and cardiac arrest. ${ }^{[3,4]}$ The risk of adverse medical events is influenced by environmental aspects such as, water and air temperature, presence of currents and accidental collisions between athletes. ${ }^{[1]}$ The cycling segment also presents environmentally-driven medical concerns. Dehydration and trauma from falling are injuries most frequently reported. Ironman distance triathlon injuries and dropouts occur mostly during the cycling and running sections respectively, most likely due to the cumulative physical and mental stress of the previous two segments, but results vary. ${ }^{[1,2]}$

There is a need for ongoing scrutiny of injury and illness profiles of ultra-distance athletes. Such data are useful for monitoring long-term changes in the frequency and circumstances of injuries, and assist greatly in the preparation of medical services. ${ }^{[5]}$

The aim of this study was to determine medical history, injury and illness profiles of athletes who received medical attention during the 2014 IMSA event, and to investigate the time and place of presentation.

\section{Methods}

This was a retrospective, cross-sectional study. The study population consisted of all the competitors who participated in the 2014 IMSA event. All athletes who required medical attention ('patients') during the event at the main medical tent and all of the medical posts or mobile units along the route were included in this study. Spectators and staff who received medical treatment were excluded.

The information used in this study was obtained from the standard documentation of medical encounters by personnel on duty at the event. Medical and demographical information of all athletes requiring medical attention was documented as standard procedure at all IMSA events. Clinical diagnoses were made and recorded by clinical staff on duty. Diagnoses were made by means of history, clinical examination, including blood pressure, heart rate, core temperature measurement, and special investigations as needed, including blood gases, glucose, urea and electrolyte measurement, urinalysis, spirometry and ECG. The completed documentation was obtained from the Race Medical Director (RMD) and the information captured on a data collection form. For the purposes of this analysis, where a final diagnosis could not be made with the available resources, the main presenting symptoms that were treated were reported. Demographic 
information for all athletes was also obtained. Weather conditions on race day were provided by the SA Weather Service. Because of incomplete retrospective data obtained from the race medical documentation in a small number of cases, patient numbers from which percentages were obtained vary slightly. The actual numbers used are reflected in the results.

All medical staff at the 2014 IMSA event were briefed prior to the event on accurate documentation of all medical encounters. In order to prevent inter-observer bias, only one author captured the data obtained from the medical records on the data collection forms.

Data were analysed by the Department of Biostatistics, Faculty of Health Sciences, University of the Free State (UFS), using SAS version 9.3. Descriptive statistics, namely, frequencies and percentages (categorical variables) and means, and standard deviations (SD) or percentiles (numerical variables) were calculated. Subgroups were compared using Fisher's exact test or chi-squared tests, with level of statistical significance set at 0.05 .

The study was approved by the Ethics Committee of the Faculty of Health Sciences, UFS (ECUFS 119/2014). Permission was obtained from the RMD of the 2014 IMSA event.

\section{Results}

\section{Demographic data}

A total of 2331 athletes started the race of which $82.0 \%$ (n $=1911)$ were male and $18.0 \%(n=420)$ female. During the event, 179 athletes $(7.7 \%)$ reported to the race medical areas for attention. This represents 76.8 medical events per 1000 race starters. There was no statistical difference between male and female athletes, with $7.4 \%(\mathrm{n}=141)$ of male athletes and $9.1 \%(\mathrm{n}=38)$ of female athletes requiring medical attention $(P=0.24)$.

The mean age of the patients was 37.7 years (range 21-63 years; SD 8.4 years). Younger athletes between the ages of 18-24 years had the highest percentage requiring medical attention $(14.7 \%)$, followed by athletes in the $25-29$ year age group $(12.8 \%)$ (Table 1$)$. Less than $10 \%$ of athletes in all other age groups required medical attention $(P=0.04)$.

\section{Race information}

The average wind speed, temperature and humidity levels were calculated for each segment of the race. The measurements for the swim segment were $3 \mathrm{~km} / \mathrm{h}, 17.3^{\circ} \mathrm{C}$ and $81.5 \%$, for the cycling segment $6.1 \mathrm{~km} / \mathrm{h}, 19.7^{\circ} \mathrm{C}$ and $84.3 \%$, and for the running segment $7.8 \mathrm{~km} / \mathrm{h}, 20^{\circ} \mathrm{C}$ and $82.3 \%$, respectively.

Information regarding the time slots during which the athletes presented is depicted in Fig. 1. The 2014 IMSA event started at 06:00 and concluded at 24:00, with total possible duration of 18 hours. The highest percentage of athletes ( $\mathrm{n}=33 ; 18.4 \%$ ) presented between $19 \mathrm{~h} 00$ and 19h59. There was an increase in patient presentation from 16:00 until approximately 23:00, during which time 88 patients $(49.2 \%)$ attended the medical facilities. The median duration of stay was 26 minutes per patient (range

\section{2-161 minutes).}

Table 2 shows the part of the race during which the athletes presented. Most patients $(\mathrm{n}=129 ; 80.1 \%$ ) presented after completing the race. The second highest number of presentations were during the cycling segmentation $(\mathrm{n}=17$; $10.6 \%)$.

\section{Pre-race medical history and medication use}

The use of chronic medication was reported by $19.5 \%(n=34)$ of patients. The most common chronic medications were asthma medications (5.2\%) and anti-hypertensives (4.0\%). Of the 179 patients, $36.2 \%(n=63)$ reported using acute medication in the three days before the race. The most common reported medications were non-steroidal anti-inflammatory medication (NSAIDs) $(\mathrm{n}=22 ; 12.1 \%)$ and analgesics $(\mathrm{n}=10 ; 5.8 \%)$. In the 30 days preceding the event, $27.8 \%(n=48)$ of the patients reported suffering from an illness. Thirty-six $(20.8 \%)$ patients reported an upper respiratory tract infection (URTI) and five $(2.9 \%)$ reported gastroenteritis.

Table 1. Biographical variable of age (years)

\begin{tabular}{cccc}
\hline & \multicolumn{2}{c}{$\begin{array}{c}\text { Athletes requiring medical attention } \\
\text { during the IMSA 2014 event }\end{array}$} & \\
\cline { 2 - 3 } Age categories & $\begin{array}{c}\text { Yes }(\mathrm{n}=174) \\
\mathrm{n}(\%)\end{array}$ & $\begin{array}{c}\text { No }(\mathrm{n}=2 \text { 157) } \\
\mathrm{n}(\%)\end{array}$ & $\begin{array}{c}\text { Total }(\mathrm{n}=2331) \\
\mathrm{n}(\%)\end{array}$ \\
\hline $18-24$ & $5(14.7)$ & $29(85.3)$ & $34(1.5)$ \\
$25-29$ & $28(12.8)$ & $191(87.1)$ & $219(9.4)$ \\
$30-34$ & $36(8.1)$ & $410(91.9)$ & $446(19.1)$ \\
$35-39$ & $33(7.5)$ & $410(92.6)$ & $443(19.0)$ \\
$40-44$ & $38(7.0)$ & $503(93.0)$ & $541(23.2)$ \\
$45-49$ & $16(4.9)$ & $308(95.1)$ & $324(13.9)$ \\
$50-54$ & $12(5.9)$ & $191(94.1)$ & $203(8.7)$ \\
$55-59$ & $4(4.6)$ & $83(95.4)$ & $87(3.7)$ \\
$60-64$ & $2(7.7)$ & $24(92.3)$ & $26(1.1)$ \\
$65+$ & $0(0)$ & $8(100.0)$ & $8(0.3)$ \\
\hline$n=$ total number of athletes, \% $=$ percentage of total athletes &
\end{tabular}

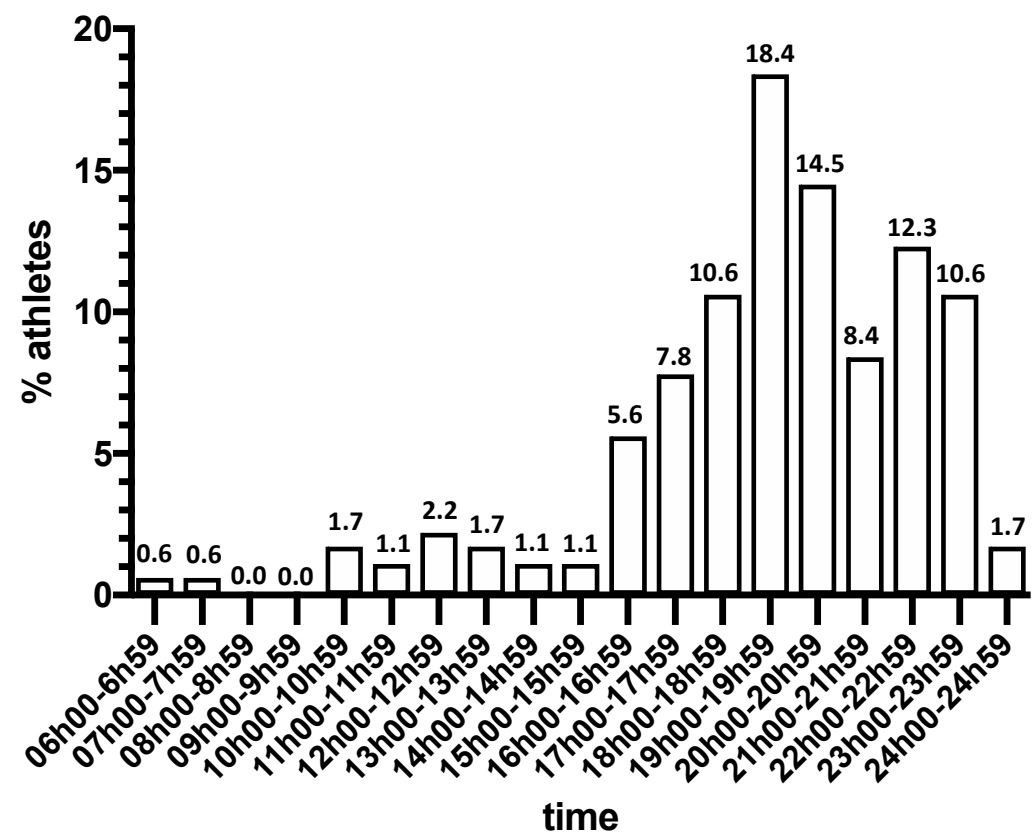

Fig 1. Distribution of medical contacts per time of day 
Table 2. Stages of the race completed by the athletes requiring medical attention during the 2014 IMSA event $(n=161)$

\begin{tabular}{ll}
\hline Stage & $\mathrm{n}(\%)$ \\
\hline Swim & $2(1.2)$ \\
Stopped at first transition & $0(0)$ \\
Stopped during cycling & $17(10.6)$ \\
Stopped at second transition & $4(2.5)$ \\
Stopped during marathon & $9(5.6)$ \\
Completed the race & $129(80.1)$ \\
\hline$n=$ total number of athletes, $\%=$ percentage of total athletes
\end{tabular}

Table 3. Medication used during the 2014 IMSA by athletes requiring medical attention $(n=61)$

\begin{tabular}{ll}
\hline Medication & n (\%) \\
\hline Non-steroidal anti-inflammatory (NSAIDs) & $28(16.1)$ \\
Analgesics & $25(14.4)$ \\
Anti-diarrheal medication & $7(4.0)$ \\
Muscle relaxants & $7(4.0)$ \\
Anti-nausea/vertigo/abdominal cramp tablets & $4(2.3)$ \\
Crampease & TM (Johannesburg, South Africa) \\
Antacids & $3(1.7)$ \\
Magnesium tablets & $3(1.7)$ \\
Salt tablets & $2(1.2)$ \\
\hline
\end{tabular}

$n=$ total number of athletes, $\%=$ percentage of total athletes

\section{Medication use during the IMSA}

More than a third of patients $(35.1 \%, \mathrm{n}=61)$ reported using medication during the race (Table 3 ). The most common medications were NSAIDs (16.1\%) and analgesics (14.4\%).

\section{Diagnoses, symptoms and signs}

Medical complaints encountered by medical staff at the IMSA were categorised in clusters, as presented in Table 4 . The most common group of conditions was exertion-related ( $\mathrm{n}=137$; $64.6 \%$ of diagnoses made): $34.0 \%$ cases of exercise-associated collapse $(\mathrm{EAC}) /$ hypotension $(\mathrm{n}=72), 9.4 \%$ dehydration $(n=20), 5.2 \%$ exhaustion $(n=11), 5.2 \%$ hypothermia $(n=11)$ and $5.2 \%$ exercise-associated muscle cramps (EAMC) $(n=11)$. Six cases of hyponatraemia were diagnosed (2.8\%). Hypoglycaemia $(n=5)$ accounted for $2.4 \%$ of cases and hyperthermia $(\mathrm{n}=1)$ accounted for $<1 \%$.

The second most prevalent cluster of diagnoses was related to the gastrointestinal system $(n=29 ; 13.7 \%)$. Sixteen cases of nausea with or without vomiting were recorded $(7.6 \%)$, followed by epigastric pain and diarrhoea $(n=6 ; 2.8 \%$ each), and one case of heartburn $(<1 \%)$. The dermatological cluster contributed $9.9 \%(\mathrm{n}=21)$, mostly skin abrasions ('road rash') ( $\mathrm{n}=10 ; 4.7 \%)$, followed by blisters $(\mathrm{n}=7 ; 3.3 \%)$, two bee stings $(<1 \%)$ and one case of erythema and sunburn $(<1 \%)$ each.

Seventeen musculoskeletal diagnoses were recorded $(8.0 \%)$ : $10(4.7 \%)$ overuse injuries (low back pain, Achilles tendon pain, peroneal tendon pain, cervical muscle spasm, patellofemoral pain, and iliotibial band friction syndrome), and seven $(3.3 \%)$ acute traumatic injuries (muscle sprains, ankle sprains, and shoulder dislocation).

Five $(2.4 \%)$ cardiorespiratory diagnoses were recorded, all potentially serious. One diagnosis of pulmonary embolism, atrial fibrillation, asthma, bronchospasm and sore throat with coughing each, were made.

\section{Special investigations}

Blood glucose testing was performed in $85.8 \%(n=152)$ of the patients, while blood gas and urea and electrolytes were tested in $29.9 \%$ of the patients, respectively $(\mathrm{n}=53)$. Electrocardiograms (ECGs) were done in three $(1.7 \%)$ patients.

\section{Discussion}

Despite the increasing popularity of Ironman distance triathlons, there is a lack of data on injury and illness profiles in these events. ${ }^{[5]}$ IMSA and other events rely on historical data collected from previous events to plan the medical facilities and services for future events. This requires data on the predicted number, nature and severity of the casualties. ${ }^{[2,5]}$

\section{Incidence of medical encounters}

Of the 2331 athletes competing, 7.7\% required medical attention. This is lower than the $10.1 \%$ documented for the 2013 IMSA event. ${ }^{[6]}$ Injury and illness rates are recorded and reported with inconsistent methodology, making results difficult to compare. There are many external event-related variables which influence medical encounter data, including environmental factors, distances, age and the level of competence of competitors. ${ }^{[1,2,7]}$ Similar studies noted a treatment presentation rate of between $2 \%$ and $37.7 \%{ }^{[2,5]}$ Medical staff can therefore estimate to manage approximately $30 \%$ of race starters during the course of the event, but can amend the estimates according to recent statistics and environmental variables on race day.

\section{Timing of medical encounters}

The majority of medical encounters (80.1\%) occurred after the race, while nearly half occurred in the last eight hours. Similar trends have been recorded in other Ironman events, with peak times of medical encounters coinciding with that of finishing times. ${ }^{[2,5]}$ A positive correlation between the time of arrival and treatment duration has been recorded previously, meaning patients arriving later are expected to require longer treatment times. ${ }^{[5]}$ Little has been published on the numbers and qualifications of medical personnel at Ironman events. A medical support team comprising a medical director, one additional physician per 200 athletes, one nurse per 100 athletes and additional paramedical and ambulance staff are recommended for a standard triathlon. ${ }^{[3]}$ The onsite medical team for the half- and Ironman distance triathlon with 1923 participants in rural Canada consisted of 120 members, of which key personnel were five physicians, 11 nurses and 36 paramedics. ${ }^{[2]}$ Rimmer and Coniglione ${ }^{[5]}$ concluded that medical care at amateur triathlon events should be planned according to the temporal flow of injuries throughout the event, and that medical support be increased by $20 \%$ to three doctors, nine nurses and support staff per 100 participants in the latter stages of triathlons. In this study, the peak patient intake was 33 patients per hour, and the average intake for the last eight hours was 20 patients per hour. The IMSA medical plan provides for a staffing schedule according to expected patient flow, allocating one doctor and two nurses per four patients. Provision is made for 37 beds of which three are intensive care 
Table 4. Diagnoses, symptoms or signs of athletes requiring medical attention

\begin{tabular}{|c|c|c|c|c|}
\hline Diagnosis cluster & Diagnosis & $\mathbf{n}^{*}$ & $\%$ of 177 patients & $\%$ of 212 diagnoses \\
\hline \multirow[t]{9}{*}{ Metabolic/Exertion-related } & & & $126(71.2)$ & $137(64.6)$ \\
\hline & $\begin{array}{l}\text { Exercise associated collapse } \\
\text { (EAC)/hypotension }\end{array}$ & 72 & 40.7 & 34.0 \\
\hline & Dehydration & 20 & 11.3 & 9.4 \\
\hline & Exhaustion & 11 & 6.2 & 5.2 \\
\hline & $\begin{array}{l}\text { Exercise-associated muscle cramps } \\
\text { (EAMC) }\end{array}$ & 11 & 6.2 & 5.2 \\
\hline & Hypothermia & 11 & 6.2 & 5.2 \\
\hline & Hyponatremia & 6 & 3.4 & 2.8 \\
\hline & Hypoglycaemia & 5 & 2.8 & 2.4 \\
\hline & Hyperthermia & 1 & 0.6 & 0.5 \\
\hline \multirow[t]{5}{*}{ Gastrointestinal } & & & $29(16.4)$ & $29(13.7)$ \\
\hline & Nausea & 16 & 9.1 & 7.6 \\
\hline & Epigastric pain & 6 & 3.4 & 2.8 \\
\hline & Diarrhoea & 6 & 3.4 & 2.8 \\
\hline & Heartburn & 1 & 0.6 & 0.5 \\
\hline \multirow[t]{6}{*}{ Dermatological } & & & 21 (11.9) & $21(9.9)$ \\
\hline & Skin abrasions ('road rash') & 10 & 5.6 & 4.7 \\
\hline & Blisters & 7 & 4.0 & 3.3 \\
\hline & Bee sting & 2 & 1.1 & 0.9 \\
\hline & Erythema & 1 & 0.6 & 0.5 \\
\hline & Sunburn & 1 & 0.6 & 0.5 \\
\hline \multirow[t]{10}{*}{ Musculoskeletal } & & & $17(9.6)$ & $17(8.0)$ \\
\hline & Lower back pain & 4 & 2.3 & 1.9 \\
\hline & Muscle strains & 3 & 1.7 & 1.4 \\
\hline & Ankle sprains & 3 & 1.7 & 1.4 \\
\hline & Achilles tendon pain & 2 & 1.1 & 0.9 \\
\hline & Peroneal tendon pain & 1 & 0.6 & 0.5 \\
\hline & Cervical muscle spasm & 1 & 0.6 & 0.5 \\
\hline & Patellofemoral pain & 1 & 0.6 & 0.5 \\
\hline & Shoulder dislocation & 1 & 0.6 & 0.5 \\
\hline & Iliotibial band friction syndrome & 1 & 0.6 & 0.5 \\
\hline \multirow[t]{6}{*}{ Cardiorespiratory } & & & $5(2.4)$ & $5(2.4)$ \\
\hline & Pulmonary embolism $t$ & 1 & 0.6 & 0.5 \\
\hline & Arial fibrillation & 1 & 0.6 & 0.5 \\
\hline & Asthma & 1 & 0.6 & 0.5 \\
\hline & Bronchospasm & 1 & 0.6 & 0.5 \\
\hline & Sore throat and coughing & 1 & 0.6 & 0.5 \\
\hline \multirow[t]{2}{*}{ Neurological } & & & $2(1.1)$ & $2(0.9)$ \\
\hline & Concussion & 2 & 1.1 & 0.9 \\
\hline \multirow[t]{2}{*}{ Unclassified } & & & $1(0.6)$ & $1(0.5)$ \\
\hline & Urine discolouration & 1 & 0.6 & 0.5 \\
\hline
\end{tabular}

$n=$ total number of athletes, ${ }^{*} A$ patient can have more than one diagnosis in a diagnosis cluster.

+Diagnosis made on recent history of pulmonary embolism, chest pain, haemoptysis and ECG changes.

and two are equipped for surgical cases. ${ }^{\left[{ }^{[8]}\right.}$ Using this formula, the maximum bed occupation in the IMSA medical facility has never exceeded 30 (Dr. Konrad Von Hagen, IMSA Medical Director, personal communication). Mobile or secondary units should be available along the cycling, running and changeover areas, and dedicated staff and equipment should be readily available on or near the water for swift management of emergencies from the swim event.

\section{Environmental factors}

The degree of environmental stress is a health risk factor in ultra-endurance events. ${ }^{[2,3]}$ One contributing factor to the relatively low incidence of medical encounters in this study is the mild weather conditions during the race. The average ambient temperature, wind strength and humidity on race day were well within the norms of the environmental recommendations for triathlons..$^{[1,3]}$

\section{Gender}

At both the 2013 and 2014 IMSA events, male patients comprised $79 \%$ and female patients $21 \%$ of the total number of athletes treated. ${ }^{[6]}$ No statistical difference between female and male medical encounter rates were found in this study $(P=$ 0.24). Similar results were recorded in the Two Oceans Ultramarathon between 2008 and 2011. ${ }^{[7]}$ Other gender-related risk data are ambiguous. ${ }^{[2,9]}$ Further studies are needed to identify possible gender-specific modifiable risk factors in ultradistance athletes.

\section{Age and experience}

Athletes between the ages of 18-24 years had a significantly higher incidence of medical encounters than older athletes. 
Even though younger age is suggestive of less experience, it does not necessarily reflect the association. However, these results correlate with the finding that less running experience is a risk factor for medical complications in ultra-marathon events. ${ }^{[9,10]}$ This finding may be explained by self-selection of older, more experienced athletes with less career injuries, that older athletes possibly focus more on participation than winning, or address injuries earlier, before they escalate. ${ }^{[9]}$ Analysis of possible preventable medical complications in less experienced athletes should be pursued further in order to introduce prevention strategies. Rule adaptations have been made, including dedicated novice starting waves to minimise psychological stress and physical risk at the start of these events. ${ }^{[1]}$

\section{Pre-race medical history and medication use}

Chronic medication use was reported by $19.5 \%$ of patients, and $27.8 \%$ suffered an illness in the 30 days before the competition. Pre-existing acute and chronic medical conditions may pose an increased risk for medical complications during endurance exercise. In this retrospective study, it is unknown if there is a difference in the prevalence of pre-race acute or chronic medical conditions between athletes who did not need medical attention and those who did. Therefore no conclusions can be drawn.

Use of acute medication in the three days before the competition was reported by $36.2 \%$ of patients, and during the race by $35.1 \%$. The prevalence of medication use among all participants is not known and relative risk cannot be calculated. However, the most commonly used medication in the three days preceding the race $(12.1 \%)$ and during the race $(16.1 \%)$ was NSAIDs. These figures are much lower than the $30.0 \%$ of athletes using NSAIDs during the 2004 New Zealand Ironman. ${ }^{[11]}$ In the 2008 Brazil Ironman, 59.9\% of a cohort of participants reported using NSAIDs in the previous three months; of these, $25.5 \%, 17.9 \%$ and $47.4 \%$ took NSAIDs the day before, immediately before and during the race, respectively. ${ }^{[12]}$ Underreporting may have occurred in this study because of the athletes' apprehension to disclose risky behaviour. A high rate of non-prescribed NSAID use and limited awareness of the risks thereof have been reported in Ironman athletes. ${ }^{[12]}$

\section{Medical conditions treated during the 2014 IMSA}

The majority of patients $(71.2 \%)$ treated during the 2014 IMSA suffered from exertion-related conditions which included EAC, dehydration and exhaustion. In the 2010 Redman Triathlon, Oklahoma, USA, dehydration accounted for $50.7 \%$ of medical encounters, while dehydration and exhaustion contributed $72 \%$ of medical encounters in the 1999 Hawail Ironman event. ${ }^{[5]}$ The incidence of EAMC (5.2\%) was much lower than the $36.1 \%$ in the 2010 Redman Triathlon ${ }^{[5]}$ and the $11 \%$ in the Two Oceans Marathon from 2008-2011, ${ }^{[10]}$ but similar to the $6 \%$ recorded in the Hawaii Ironman. ${ }^{[2]}$ It seems that EAMC is more prevalent in shorter, faster events. ${ }^{[13,14]}$ Hypothermia (5.2\%) was more prevalent than hyperthermia. Low numbers of temperature regulatory dysfunction have been reported in ultra-distance events. ${ }^{[2,10]}$ Nevertheless, proper management protocols for these potentially dangerous conditions should be in place. ${ }^{[3]}$ Likewise, hyponatremia $(2.8 \%)$ and hypoglycaemia $(2.4 \%)$ were uncommon, but should be managed optimally.

Gastrointestinal system complaints were the second most common group of conditions recorded. Non-specific diagnoses of nausea were made in $7.6 \%$ of patients. It can be challenging to make specific diagnoses of a general symptom, such as nausea, in a pre-hospital setting. Serious gastrointestinal conditions, such as gastrointestinal bleeding and ischaemia, have been recorded in ultra-endurance events. ${ }^{11,2]}$ Prompt referral should be done in persistent cases, or where systemic symptoms indicate more serious disease.

The majority of dermatological (9.9\%) and musculoskeletal $(8.0 \%)$ conditions treated during the 2014 IMSA were minor, as reported previously. ${ }^{[9,10]}$ Skin conditions consisted mostly of abrasions and blisters. Only one case of sunburn was reported. This may represent underreporting as athletes may have opted not to seek treatment for sunburn. Ultraviolet radiation was measured in three athletes in the 1999 Hawaii Ironman. Mean ultraviolet radiation exposure during the competition was 8.3 times the minimal erythema doses, and greatly in excess of the International Radiation Protection Agency Guidelines, despite using sun protection factor $25+$ sunscreen. ${ }^{[15]}$ Copious amounts of sunscreen were made available to all participants during the IMSA 2014.

The number of cardiorespiratory diagnoses was low (2.4\%), but included potentially life-threatening conditions such as pulmonary emboli and atrial fibrillation. Such conditions should not be misdiagnosed for the much more common exertion-related diagnoses that can present in a similar way. Serious cardiorespiratory complications have been recorded in ultra-endurance events, ${ }^{[5,10]}$ and medical facilities should be equipped to manage such emergencies.

\section{Special investigations}

The availability of basic special investigations and ECG were essential in the diagnosis and management of a high percentage of patients during IMSA 2014. These aids are potentially lifesaving and should be standard equipment available in ultraendurance events.

\section{Conclusion}

In this study, accurate reporting of all patient encounters during the 2014 IMSA was achieved. An accurate diagnostic profile, temporal presentation and consideration of factors that could potentially influence the profile of medical encounters allowed these authors to present an approach to planning of medical resources for future IMSA competitions. In accordance with previous studies, exertion-related medical conditions have been predominant in IMSA events. Potential preventative measures have been identified, including education on potentially harmful medication use during the race, recent illness, and prevention of medical complications in younger participants.

Longitudinal prospective recording of medical encounters and associated factors, with standardised diagnostic criteria and terminology is recommended for ultra-endurance events to accumulate accurate evidence on which to plan 
prevention and medical services.

\section{Limitations}

Limitations are, however, that data on all participants could not be obtained for comparison and risk calculation. Furthermore, the specific nomenclature of conditions and standardisations of diagnoses could not be done for a more accurate comparison with the literature, as diagnoses were obtained retrospectively from the clinical notes of medical staff on duty at the event. The relative lack of recent studies on the topic necessitated the use of older comparative literature. The severity of medical conditions was not recorded.

Acknowledgements: Ms T Mulder, medical editor, School of Medicine, UFS, for technical and editorial preparation of the manuscript.

\section{Conflict of Interest and source of funding: None}

\section{References}

1. Dallam GM, Jonas S, Miller TK. Medical considerations in triathlon competition: recommendations for triathlon organisers, competitors and coaches. Sports Med 2005;35(2):143-161. [doi:. 10.2165/00007256-200535020- 00004]

2. Turris SA, Lund A, Bowles RR, et al. Patient presentations and medical logistics at full and half Ironman distance triathlons. Curr Sports Med Rep 2017;16(3):137-143. [doi:10.1249/JSR.0000000000000367]

3. The Triathlon Canada Medical Committee. Triathlon medical manual. Canada: Triathlon Canada Medical Committee, July 2000.

http://www.triathlon.mb.ca/admin/images/Seasons/sancti on_triathloncanada_medicalmanual.pdf (accessed 26 September 2016).

4. Laird RH, Johnson D. The medical perspective of the Kona Ironman Triathlon. Sports Med Arthrosc Rev 2012;20(4):239. [doi: 10.1097/JSA.0b013e3182736e8e]

5. Rimmer T, Coniglione T. A temporal model for nonelite triathlon race injuries. Clin J Sport Med 2012;22(3):249-253. [doi: 10.1097/JSM.0b013e318249945b]
6. Ironman South Africa. Statistics of the 2013 event. Internal Document. Ironman South Africa, 2013.

7. Schwabe K, Schwellnus MP, Derman W, et al. Less experience and running pace are potential risk factors for medical complications during a $56 \mathrm{~km}$ road running race: a prospective study in 26354 race starters--SAFER study II. Br J Sports Med 2014;48(11):905-911. [doi: 10.1136/bjsports- 2014093471]

8. Standard Bank IRONMAN African Championship. Medical Plan - 29 March 2015 (updated 5 March 2015). Internal Document. Ironman South Africa, 2016.

9. Krabak BJ, Waite B, Schiff MA. Study of injury and illness rates in multiday ultramarathon runners. Med Sci Sports Exerc 2011;43(12):2314-2320. [doi: 10.1249/MSS.0b013e318221bfe3]

10. Schwabe K, Schwellnus M, Derman W, et al. Medical complications and deaths in 21 and $56 \mathrm{~km}$ road race runners: a 4-year prospective study in 65865 runners--SAFER study I. Br J Sports Med 2014;48(11):912-918. [doi: 10.1136/bjsports2014-093470]

11. Wharam PC, Speedy DB, Noakes TD, et al. NSAID use increases the risk of developing hyponatremia during an Ironman triathlon. Med Sci Sports Exerc 2006;38(4):618-622. [doi: 10.1249/01.mss.0000210209.40694.09]

12. Gorski T, Cadore EL, Pinto SS, et al. Use of NSAIDs in triathletes: prevalence, level of awareness and reasons for use. Br J Sports Med 2011;45(2):85-90. [doi: 10.1136/bjsm.2009.062166]

13. Schwellnus MP, Drew N, Collins M. Increased running speed and previous cramps rather than dehydration or serum sodium changes predict exercise-associated muscle cramping: a prospective cohort study in 210 Ironman triathletes. Br J Sports Med 2011;45(8):650-656. [doi:10.1136/bjsm.2010.078535]

14. Schwellnus MP, Allie S, Derman W, et al. Increased running speed and pre-race muscle damage as risk factors for exercise-associated muscle cramps in a $56 \mathrm{~km}$ ultramarathon: a prospective cohort study. Br J Sports Med 2011;45(14):1132-1136. [doi:10.1136/bjsm.2010.082677]

15. Moehrle M. Ultraviolet exposure in the Ironman triathlon. Med Sci Sports Exerc 2001;33(8):1385-1386. [doi: 10.1097/00005768-200108000-00021] 